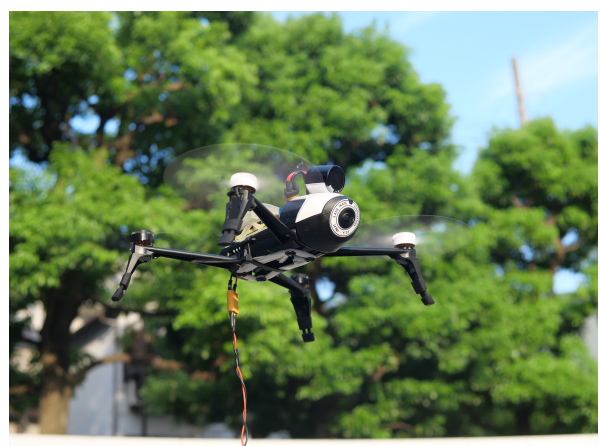

Figure 1: Flying tethered drone with prolonged flight time

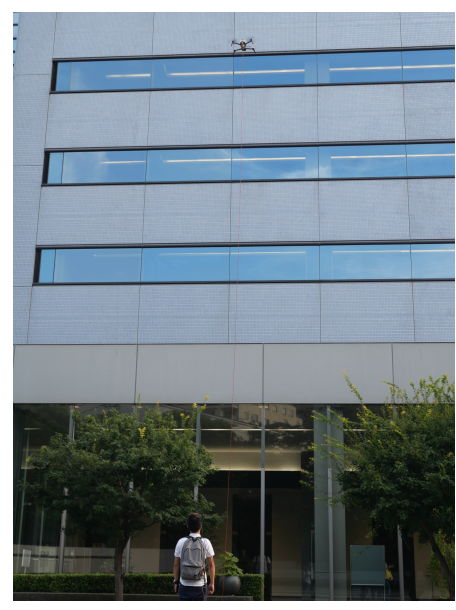

Figure 2: Extending User's Vision

\title{
Falconer: A Tethered Aerial Companion for Enhancing Personal Space
}

\section{Romain Nith}

McGill University / Sony Computer Science

Laboratories, Inc

Montreal, Canada

romain.nith@mail.mcgill.ca

\author{
Jun Rekimoto \\ The University of Tokyo / Sony Computer \\ Science Laboratories, Inc \\ Tokyo, Japan \\ rekimoto@acm.org
}

\begin{abstract}
With the growing popularity of drones, we start to see more wearable drone concepts. For the user to carry around a drone, it must be lightweight as well as have a small portable form factor. However, these constraints affect the battery capacity and therefore decrease the flight time of the vehicle. With Aerial Tethered Companion, a bigger battery is installed in the user's backpack allowing to extend significantly the flight time. Moreover, without an on-board battery, the quadcopter can carry more payload. Such system can be used in various scenarios for example in sports augmentation where the user would see itself through the drone's camera. Furthermore, Aerial Tethered Companion can be applied in telepresence where an external user would be able to see and navigate around the local user.
\end{abstract}

\section{CCS CONCEPTS}

- Human-centered computing $\rightarrow \mathrm{HCl}$ theory, concepts and models.

This paper is published under the Creative Commons Attribution 4.0 International (CC-BY 4.0) license. Authors reserve thei rights to disseminate the work on their personal and corporate Web sites with the appropriate attribution.

iHDI '19 - International workshop on Human-Drone Interaction, CHI '19 Extended Abstracts, May 5, 2019, Glasgow, Scotland, UK, http://hdi.famnit.upr.si

(c) 2019 Creative Commons CC-BY 4.0 License. 


\section{KEYWORDS}

Quadcopter, Drone, UAV,Tethered Vehicle, Augmented Perception, Augmented Sports, Telepresence

\section{ACM Reference Format:}

Romain Nith and Jun Rekimoto. 2019. Falconer: A Tethered Aerial Companion for Enhancing Personal Space. In ACM, New York, NY, USA, 6 pages.

\section{INTRODUCTION AND BACKGROUND}

Drones are becoming more and more popular these days. Unfortunately, the flight time is limited by the batteries'capacity. Therefore, to extend the flight time, the operators must choose to either change and charge multiple batteries or carry a bigger capacity battery with the cost of decreasing the weight of the payload. To keep the aerial vehicle in the sky without interruption and maximize the payload, companies like Elistair and Powerline developed tethered solutions. The idea is to decouple the battery from the chassis to have a lighter vehicle but also to increase its flight time significantly compared to current products (around 21min with at DJI Mavic Pro).

Most of the applications are aimed towards professional usage such as filmography, surveillance or exploration. However, Falconer aims to apply the tethered drone technology for human enhancement. DroneNavigator [1] already utilizes tethered drones to guide visually impaired travelers but also to extend the battery life. Whereas Flying Head [7], Drone-Augmented Human Vision [4] and Multi-View Augmented Reality with a Drone [8] show how drones can augment human vision by exploring places where the user could not go or see. Additionally, with tele-operating capabilities, Falconer allows virtual users to explore new locations.

Figure 3: Power Unit of Tethered Drone

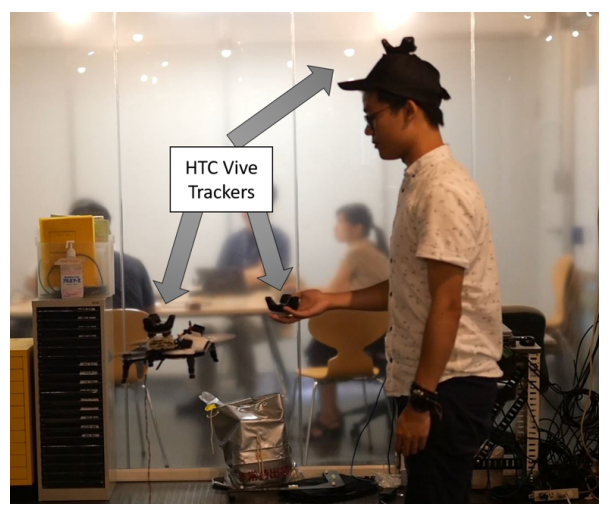

Figure 4: HTC Vive Motion Capture Setup

\section{TETHERED DRONE}

For a wearable drone, the main criteria are weight and size. The Parrot's Bebop 2 falls into this category as it is small and light enough to be carried around but also delivers enough power to fly outdoors to counter drifts. Additionally, with its GPS and ground-facing camera, the UAV (Unmanned Aerial Vehicle) can maintain its position accurately via Parrot's low-level software.

\section{Power Unit}

As designed for outdoor usage, the Bebop 2 can peak up to $480 \mathrm{~W}$ at full throttle. In this scenario to avoid having a heavy cable disturbing the balance of the drone, we decreased the current by increasing the voltage in the power delivery cable. Therefore, the power system is composed of two elements: a $48 \mathrm{~V}$ power source and a $48 \mathrm{~V}$ to $12 \mathrm{~V}$ DC-DC converter as shown in Figure 3. Since the drone is capable of pulling $10 \mathrm{~A}$ at $48 \mathrm{~V}$, we chose to use $20 \mathrm{AWG}$ wires allowing a maximum of $11 \mathrm{~A}$ to flow through the cable safely according to the American wire gauge table. Furthermore, for our application we will 


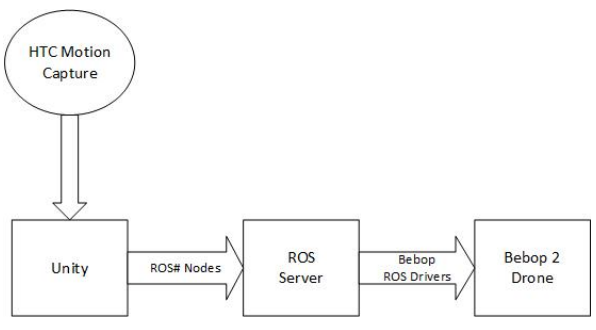

Figure 5: Manual Control System Architecture

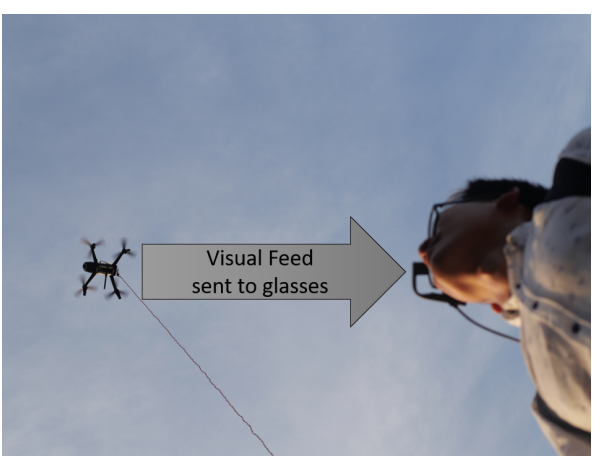

Figure 6: User's Vision Extended by Drone's Camera Through AR Glasses

${ }^{1}$ ROS Sharp by Siemens: https://github.com/siemens/ros-sharp

${ }^{2}$ bebop_autonomy - ROS Driver for Parrot Bebop Drone (quadcopter) $1.0 \& 2.0$ : https://bebopautonomy.readthedocs.io/en/latest/index.html only be deploying $5 \mathrm{~m}$ of cable, but the system can easily be scaled up to $16 \mathrm{~m}(33.31 \mathrm{~m} \Omega / \mathrm{m})$. With the different proposed applications, the power source can vary from a wall outlet through a 48V AC-DC power supply or directly at $48 \mathrm{~V}$ battery pack. With a capacity of $10 \mathrm{Ah}$, we can expect around $1 \mathrm{~h} 15 \mathrm{~min}$ of flight time if we assume a constant current draw of $8 \mathrm{Ah}$.

\section{Tracking}

We used HTC Vive Trackers mounted on the drone, the user's head and hand to track each other's position. The head tracker is used for user 's location and orientation whereas the hand tackers detect gestures to control the drone. As the HTC Vive system needs IR transmitters called Lighthouses, tracking can only be done indoors at the current state.

\section{CONTROLS}

\section{System}

The main tools used are Unity and ROS. Unity takes care of all the controls using the tracking information gathered by the HTC Vive Trackers and sends it to a ROS server through nodes using Siemens' ROS\# ${ }^{1}$ Unity plugin. Finally, the server communicates to the Bebop 2 drone through bebop_autonomy's ${ }^{2}$ ROS driver.

\section{Gesture Control}

Human tend to interact with drones like the would with another individual or a pet[2]. A combination of both gesture and voice commands could add various type of control over the drone. However, voice recognition might be hard to detect in an outdoor environment, especially with the loudness of the current generation of drones. Therefore, a simple User Interface illustrates how the user can interact with the drone using only gestures. When the drone is grounded, the user flips its tracked hand, palm facing up, to takeoff the vehicle. The latter will make its way to the front of the operator by circling around him/her depending on where it is located. He/she can then move in any direction, the drone will follow by maintaining the same distance and orientation according to the user. To trigger gesture control, the operator raises its tracked hand above hip level and can then move the drone similarly to a joystick approach. By bringing the hand back below hip level, the UAV will remain in its the last known position relative to the user, even when the latter is moving. Finally, to land the drone, the operator keeps his/her hand with the palm pointing the sky but brings it behind his/her back.

\section{Follow Me}

Ideally, we would use solely the drone 's camera to track and follow the user. Flying Eyes [6] proposed a system using computer vision but distance estimation was inaccurate. By combining accurate GPS 


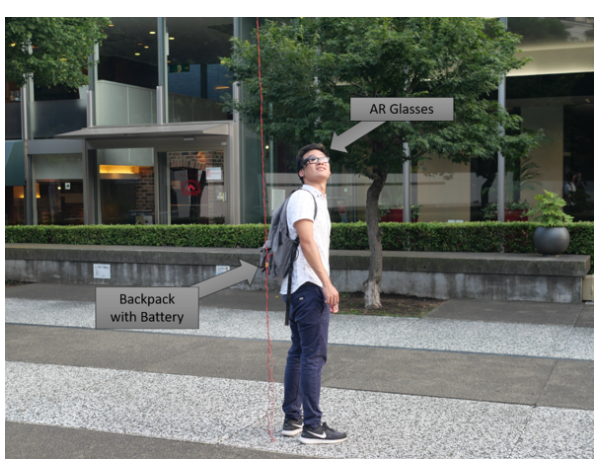

Figure 7: User equipped with AR glasses and battery backpack

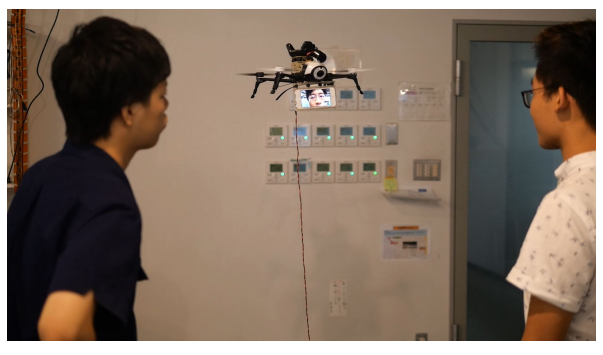

Figure 8: Telepresence video conference with external user coordinates from the two points of interest, we can increase the estimation but only on a 2D plane. Therefore we added a barometer alongside the GPS sensor to extract the altitude. Using a simple projection, we can find the respective distance between the drone and the user. However, the proposed solution is greatly limited by the accuracy of the sensors and did not output reliable data. However, DroneTrack [10] uses acoustic sounds to locate and track the user. Unfortunately, this solution is only ideal for close environments usage as there are too many noise disturbance in the outdoors.

\section{APPLICATIONS}

\section{Perception Augmentation}

Falconer is a personal companion that augments the user's abilities, more specifically in this scenario, its vision. Since the Parrot drone is equipped with an on-board camera, the live feed can be easily extracted. With an extra pair of eyes in the skies, the quadcopter provides information of the surrounding through a pair of augmented reality glasses to maximize the user's visibility. Figure 6 illustrates how the user can use Falconer to see the horizon from a higher point of view.

\section{Sports Augmentation}

The Flying Eyes [6] research showcases an innovative method of training for a sport where the user see itself in a third person view. Being able to see one 's self when practicing an activity helps highlight body motions and optimize each movement. In a similar fashion, dancers already apply the technique in front of a mirror. Furthermore, a third person view training appears to have no diminishing results compared to the normal perspective $[3,12]$. On the contrary, a third person perspective does enhance one 's performance when the activity involves the need for surrounding knowledge. In rock climbing, the climber has a restricted view to what is above him/her. With an out-of-body view, Falconer can aid choosing the most optimized path. Therefore, through Falconer's eyes, an out-of-body experience helps athlete with various training as well as act as a tool to help athlete make better choices to improve performance.

\section{Engaging Telepresense}

Not having the battery on the drone permits to carry a heavier payload. Attaching a screen or a phone onto the Bebop 2 opens many possibilities for telepresence. Scalable Body [11] attaches a camera on a scalable pole to a movable robotized base. This allows to reflect more accurately the remote operator 's height while also piloting the robot. Falconer reproduces the same effect but with a drone. The remote user can operate the drone to explore and experience the same landscape as the local users. For indoor usage, the grounded power source can either be directly connected to an outlet, providing 
unlimited flight time but limited mobility within the range of the wire. Whereas for outdoors, like previously mentioned, the battery pack can be stored in the user's backpack.

\section{DISCUSSION}

The current state of Falconer is quite limited at the moment. The different topics outlined through out the paper shows how versatile a tethered drone can be.

With the current prototype, the cable linking the drone to the power supply can not vary its length, meaning as the vehicle flies closer to the user, unnecessary wires are left on the floor. In the near future, we would like to implement a system that would wind back the cable to minimize the tension as well as the length of the wire left between the drone and the battery.

As we want to implement a relative position control [5] to be able to control the UAV precisely without hindering the experience, other type of tracking should be considered such depth sensing cameras or SLAM. Additionally, by knowing how much wire has been released, we could calculate the distance separating the drone and the user. It would therefore give us more information to help maneuvering the vehicle autonomously around the user. With a decent localization system, the wearable drone would be able to self-takeoff and self-park onto the user's backpack with great accuracy.

Without a battery on-board, the system completely relies on the connection from the power source to the quadcopter. Adding a small capacity battery can prevent disastrous crashes if the main power system fails. If the capacity allows it, implementing a similar design as Evercopter [9] could widen the possibilities of Falconer. The drone would connect to the power source through a magnetic link and would be able to disconnect itself and fly-out independently for a short among of time.

\section{CONCLUSIONS}

Tethering a drone greatly opens new applications for professional uses. However, in this paper, we explored how such system can be beneficial to an individual. Falconer is a prototype of a platform for a personal aerial companion. We dived into how it can enhance one 's personal space by augmenting the human vision as well as bringing closer humans with more engaging interactions through telepresence. In the next iteration, we will be addressing the different key components discussed in the discussion section along with refining the design of the current system.

\section{REFERENCES}

[1] Mauro Avila, Markus Funk, and Niels Henze. 2015. DroneNavigator: Using Drones for Navigating Visually Impaired Persons. In Proceedings of the 17th International ACM SIGACCESS Conference on Computers \&\#38; Accessibility (ASSETS 15). ACM, New York, NY, USA, 327-328. https://doi.org/10.1145/2700648.2811362

[2] Jessica R. Cauchard, Jane L. E, Kevin Y. Zhai, and James A. Landay. 2015. Drone \&\#38; Me: An Exploration into Natural Human-drone Interaction. In Proceedings of the 2015 ACM International Joint Conference on Pervasive and Ubiquitous Computing (UbiComp '15). ACM, New York, NY, USA, 361-365. https://doi.org/10.1145/2750858.2805823 
[3] Alexandra Covaci, Anne-Hélène Olivier, and Franck Multon. 2014. Third Person View and Guidance for More Natural Motor Behaviour in Immersive Basketball Playing. In Proceedings of the 20th ACM Symposium on Virtual Reality Software and Technology (VRST '14). ACM, New York, NY, USA, 55-64. https://doi.org/10.1145/2671015.2671023

[4] Okan Erat, Werner Alexander Isop, Denis Kalkofen, and Dieter Schmalstieg. 2018. Drone-Augmented Human Vision: Exocentric Control for Drones Exploring Hidden Areas. IEEE Transactions on Visualization and Computer Graphics 24, 4 (April 2018), 1437-1446. https://doi.org/10.1109/TVCG.2018.2794058

[5] Markus Funk. 2018. Human-drone Interaction: Let's Get Ready for Flying User Interfaces! Interactions 25, 3 (April 2018), 78-81. https://doi.org/10.1145/3194317

[6] Keita Higuchi, Yoshio Ishiguro, and Jun Rekimoto. 2011. Flying Eyes: Free-space Content Creation Using Autonomous Aerial Vehicles. In CHI '11 Extended Abstracts on Human Factors in Computing Systems (CHI EA '11). ACM, 561-570 https://doi.org/10.1145/1979742.1979627

[7] Keita Higuchi and Jun Rekimoto. 2013. Flying Head: A Head Motion Synchronization Mechanism for Unmanned Aerial Vehicle Control. In CHI '13 Extended Abstracts on Human Factors in Computing Systems (CHI EA '13). ACM, 2029-2038 https://doi.org/10.1145/2468356.2468721

[8] Aaron Hitchcock and Kelvin Sung. 2018. Multi-view Augmented Reality with a Drone. In Proceedings of the 24th ACM Symposium on Virtual Reality Software and Technology (VRST '18). ACM, New York, NY, USA, Article 108, 2 pages. https://doi.org/10.1145/3281505.3283397

[9] Yutaro Kyono, Takuro Yonezawa, Hiroki Nozaki, Masaki Ogawa, Tomotaka Ito, Jin Nakazawa, Kazunori Takashio, and Hideyuki Tokuda. 2013. EverCopter: Continuous and Adaptive Over-the-air Sensing with Detachable Wired Flying Objects. In Proceedings of the 2013 ACM Conference on Pervasive and Ubiquitous Computing Adjunct Publication (UbiComp '13 Adjunct). ACM, New York, NY, USA, 299-302. https://doi.org/10.1145/2494091.2494183

[10] Wenguang Mao and Lili Qiu. 2018. DroneTrack: An Indoor Follow-Me System Using Acoustic Signals. GetMobile: Mobile Comp. and Comm. 21, 4 (Feb. 2018), 22-24. https://doi.org/10.1145/3191789.3191798

11] Akira Matsuda, Takashi Miyaki, and Jun Rekimoto. 2017. ScalableBody: A Telepresence Robot That Supports Face Position Matching Using a Vertical Actuator. In Proceedings of the 8th Augmented Human International Conference (AH '17). ACM New York, NY, USA, Article 13, 9 pages. https://doi.org/10.1145/3041164.3041182

[12] Patrick Salamin, Tej Tadi, Olaf Blanke, Frederic Vexo, and Daniel Thalmann. 2010. Quantifying Effects of Exposure to the Third and First-Person Perspectives in Virtual-Reality-Based Training. IEEE Trans. Learn. Technol. 3, 3 (July 2010), 272-276. https://doi.org/10.1109/TLT.2010.13 